\title{
Monitoring Out-of-State Patients during a 2017 Hurricane Response using ESSENCE
}

\author{
Caleb Wiedeman*1, Julie Shaffner², Kelly Squires ${ }^{1}$, Jeffrey Leegon ${ }^{1}$, Rendi Murphree $^{2}$ \\ and Paul E. Petersen ${ }^{1}$
}

${ }^{1}$ Tennessee Department of Health, Nashville, TN, USA; ${ }^{2}$ Centers for Disease Control and Prevention, Atlanta, GA, USA

\section{Objective}

To demonstrate the use of ESSENCE in the BioSense Platform to monitor out-of-State patients seeking emergency healthcare in Tennessee during Hurricanes Harvey and Irma.

\section{Introduction}

Syndromic surveillance is the monitoring of symptom combinations (i.e., syndromes) or other indicators within a population to inform public health actions. The Tennessee Department of Health (TDH) collects emergency department (ED) data from more than 70 hospitals across Tennessee to support statewide syndromic surveillance activities. Hospitals in Tennessee typically provide data within 48 hours of a patient encounter. While syndromic surveillance often supplements disease- or condition-specific surveillance, it can also provide general situational awareness about emergency department patients during an event or response.

During Hurricanes Harvey (continental US landfall on August 25, 2017) and Irma (continental US landfall on September 10, 2017), TDH supported all hazards situational awareness using the Electronic Surveillance System for the Early Notification of Community-based Epidemics (ESSENCE) in the BioSense Platform supported by the National Syndromic Surveillance Program (NSSP). The volume of out-of-state patients in Tennessee was monitored to assess the impact on the healthcare system and any geographic- or hospital-specific clustering of out-of-state patients within Tennessee. Results were included in daily State Health Operations Center (SHOC) situation reports and shared with agency response partners such as the Tennessee Emergency Management Agency (TEMA).

\section{Methods}

Data were monitored from August 18, 2017 through September 24, 2017. A simple query was established in ESSENCE using the Patient Location (Full Details) dataset. Data were limited to hospital ED visits reported by Tennessee (Site = "Tennessee"). To monitor ED visits among residents of Texas before, during, and after Major Hurricane Harvey, data were queried for a patient zip code within Texas (State $=$ "Texas"). ED visits among Florida residents were monitored similarly (State = "Florida") before, during, and after Major Hurricane Irma. Additionally, a free text chief complaint search was implemented for the terms "Harvey", "Irma, "hurricane", "evacuee", "evacuate", "Florida", and "Texas". Chief complaint search results were then filtered to remove encounters with patient zip codes within Tennessee.

\section{Results}

From August 18, 2017 through September 24, 2017, Tennessee hospital EDs reported 277 patient encounters among Texas residents and 1,041 patient encounters among Florida residents. The number of encounters among patients from Texas remained stable throughout the monitoring period. In contrast, the number of encounters among patients from Florida exceeded the expected value on September 7, peaked September 10 at 116 patient encounters, and returned to expected levels on September 16 (Figure 1). The increase in patients from Florida was evenly distributed across most of Tennessee, with some clustering around a popular tourism area in East Tennessee.
No concerning trends in reported syndromes or chief complaints were identified among Texas or Florida patients.

The free text chief complaint query first exceeded the expected value on September 9, peaked on September 11 with 5 patient encounters, and returned to expected levels on September 14. From August 18 through September 24, 21 of 30 visits captured by the query were among Florida residents. One Tennessee hospital appeared to be intentionally using the term "Irma" in their chief complaint field to indicate patients from Florida impacted by the hurricane.

\section{Conclusions}

The ESSENCE instance in the BioSense platform provided TDH the opportunity to easily locate and monitor out-of-state patients seen in Tennessee hospital EDs. While TDH was unable to validate whether all patients identified as residents of Florida were displaced because of Major Hurricane Irma, the timing of the rise and fall of patient encounters was highly suggestive. Likewise, seeing no substantial increase ED patients with residence in Texas reassured TDH that the effects of Hurricane Harvey were not impacting hospital emergency departments in Tennessee.

TDH used information and charts from ESSENCE to support situational awareness in our SHOC and at TEMA. Use of patient zip code to identify out-of-state residents was more sensitive than chief complaint searches by keyword during this event. ESSENCE allowed TDH to see where out-of-state patients appeared to be concentrating in Tennessee and monitor the need for targeting messaging and resources to heavily affected areas. Additionally, close surveillance of chief complaints among out-of-state patients provided assurance that no unusual patterns in illness or injury were occurring.

ESSENCE is the only TDH information source capable of rapidly collecting health information on out-of-state patients. ESSENCE allowed TDH to quickly identify a change within the patient population seen at Tennessee emergency departments and monitor the situation until the patient population returned to baseline levels.

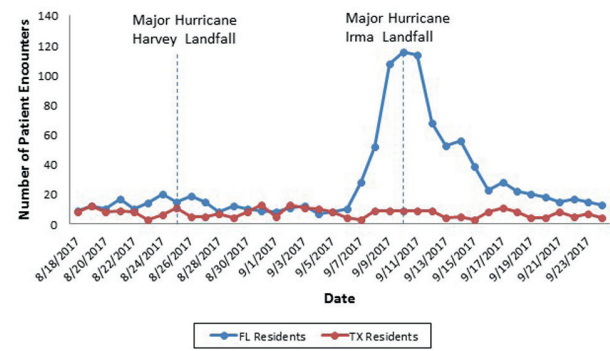

Figure 1: Emergency department (ED) encounters reported among patient with residence in Texas and Florida, August 18, 2017 - September 24, 2017.

\section{Keywords}

ESSENCE; BioSense; Emergency Preparedness; Syndromic surveillance

\section{${ }^{*}$ Caleb Wiedeman}

E-mail: caleb.wiedeman@tn.gov 